\title{
Malformações Fetais em Gestação Múltipla
}

\author{
Fetal Malformations and Multiple Pregnancy
}

\begin{abstract}
Maria de Lourdes Brizot ${ }^{1}$, Marisa Marie Fujita ${ }^{1}$, Nádia Stela Viegas dos Reis ${ }^{1}$, Jorge Demétrio Banduki Neto ${ }^{1}$, Regina Schultz ${ }^{2}$, Seizo Miyadahira ${ }^{1}$, Marcelo Zugaib ${ }^{1}$
\end{abstract}

\section{RESUM0}

Objetivos: demonstrar os tipos de malformações em gestações múltiplas assim como a influência da corionicidade nestes casos.

Métodos: foram analisadas 169 gestações múltiplas submetidas à avaliação ultra-sonográfica no Setor de Medicina Fetal da Clínica Obstetricia do Hospital das Clinicas da Faculdade de Medicina da Universidade de São Paulo no periodo de janeiro de 1997 a janeiro de 1999. A corionicidade foi definida por meio de ultra-sonografia no primeiro trimestre, presença de placentas separadas, sexos diferentes ou exame anatomopatológico da placenta.

Resultados: do total de gestações, 24 apresentavam malformações fetais (14,2\%) sendo 22 em gestações duplas e 2 em gestações triplas. Das gestações gemelares que apresentavam malformações fetais, 13 eram monocoriônicas, 4 dicoriônicas e em 5 a corionicidade não era conhecida. Os tipos de malformações fetais foram aquelas associadas unicamente a gestações múltiplas (gêmeos unidos, $n=5$; gêmeo acárdico, $n=3$ ) e as que também ocorrem em gestações únicas. A idade gestacional do parto de fetos com anomalias foi menor em relação aos sem anomalias.

Conclusões: a maioria das malformações congênitas ocorreu em gestações monocoriônicas. A determinação precoce do tipo de placentação é útil no estabelecimento do prognóstico de gestações gemelares, permitindo o planejamento das condutas em gestações complicadas por anomalias congênitas.

PALAVRAS-CHAVE: Gestação múltipla. Malformações fetais. Ultra-sonografia. Placenta. Diagnóstico pré-natal.

\section{Introdução}

Gêmeos e anomalias congênitas são alvo de pesquisa há séculos. Em particular, o estudo de gêmeos tem sido utilizado para elucidar a contribuição de fatores genéticos à variação fenotípica normal, ao comportamento e a muitas doenças. Além disso, a incidência de malforma-

\footnotetext{
${ }^{1}$ Setor de Medicina Fetal da Clínica Obstétrica do Hospital das Clínicas da Faculdade de Medicina da Universidade de São Paulo.

${ }^{2}$ Divisão de Anatomia Patológica do Hospital das Clínicas da Faculdade de Medicina da Universidade de São Paulo.

Correspondência:

Maria de Lourdes Brizot

Departamento de Obstetrícia e Ginecologia do Hospital das Clínicas da Faculdade de Medicina da Universidade de São Paulo.

Instituto Central, $10^{\circ}$ Andar Sala 10083

Rua Dr. Enéas de Carvalho Aguiar 255 - Cerqueira César

05403-000 - São Paulo - SP

e-mail: mlbrizot@uol.com.br
}

ções congênitas é consideravelmente aumentada em gestações múltiplas comparada a gestações únicas ${ }^{1-4}$. A ocorrência de malformações maiores em crianças gêmeas é de $2 \%$, o dobro em relação a crianças provenientes de gestações únicas ${ }^{5}$. O diagnóstico de anomalias fetais em gestações múltiplas pode ser realizado durante o pré-natal por meio do exame ultra-sonográfico. Neste exame é possivel detectar anomalias de um ou ambos os fetos ou, inversamente, excluir alterações específicas cujo risco é conhecidamente aumentado em gêmeos.

Há condições especialmente únicas em gêmeos que contribuem para o aumento da morbidade e mortalidade perinatal. Estas condições incluem: gêmeos monoamnióticos, anomalias congênitas exclusivas de gêmeos, óbito fetal intra-útero e síndrome da transfusão feto-fetal. A determinação pré-natal da corionicidade pela ultra-sonografia, no primeiro trimestre da gesta- 
ção, é a chave para o diagnóstico e conduta em face de muitas destas condições. A definição da corionicidade também é importante para o planejamento das condutas de gestações gemelares complicadas por crescimento e anomalias congênitas discordantes.

Este estudo tem o propósito de demonstrar os tipos de malformações em gestações múltiplas, assim como a influência da corionicidade nestes casos.

\section{Pacientes e Métodos}

O estudo consistiu em uma análise retrospectiva de 169 casos de gestações múltiplas submetidas à avaliação ultra-sonográfica no Setor de Medicina Fetal da Clínica Obstetrícia do Hospital das Clínicas da Faculdade de Medicina da Universidade de São Paulo no período de janeiro de 1997 a janeiro de 1999. Os parâmetros da avaliação ultra-sonográfica, biometria e morfologia fetais foram obtidos e revistos do programa dBase. Os dados referentes ao parto e ao recém-nascido foram obtidos das pacientes por meio de carta resposta, entregue durante o exame, consulta ao prontuário ou contato telefônico.

Todas as gestantes foram acompanhadas com exame ultra-sonográfico mensal que consistia na avaliação: da morfologia e crescimento dos fetos; da placenta e anexos; do volume de líquido amniótico; da apresentação dos fetos e avaliação do comprimento da cérvice uterina.

Para definir a corionicidade utilizamos os seguintes parâmetros: (1) no primeiro trimestre (idade gestacional $<14$ semanas), quando a massa placentária era única, a presença do sinal de lambda (projeção do córion em direção à membrana amniótica) definia uma gestação dicoriônica (Figura 1), e a ausência deste sinal, gestação monocoriônica (Figura 2); (2) presença de placentas separadas; (3) sexos diferentes; (4) exame anatomopatológico da placenta.

\section{Resultados}

A incidência da gestação múltipla foi de $1,9 \%$ do total de avaliações ultra-sonográficas realizadas no mesmo período (169 gestações múltiplas para 9000 gestações únicas).

Das 169 gestações múltiplas, 93\% $(\mathrm{n}=157)$ eram duplas, $6 \%(\mathrm{n}=10)$ triplas e $1 \%(\mathrm{n}=2)$ quádruplas. Do total de gestações, $87 \%$ ocorreram espontaneamente conforme a Tabela 1.

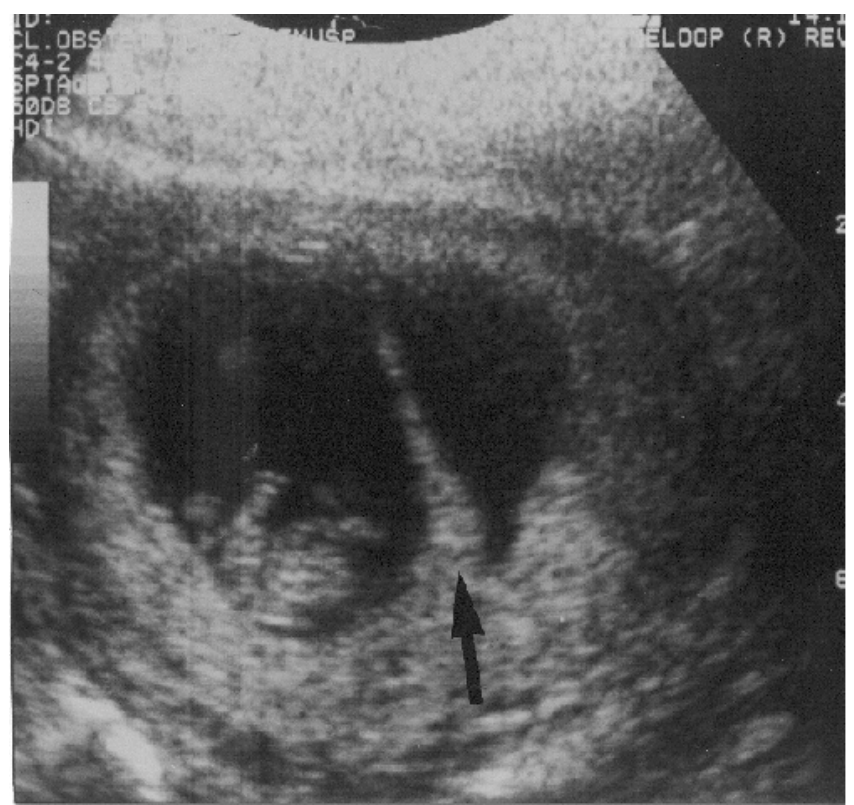

Figura 1 - Determinação da corionicidade em gestação gemelar no primeiro trimestre: massa placentária única e presença do sinal de lambda. Observar a projeção do córion em direção à membrana amniótica (seta), característico da gestação dicoriônica.

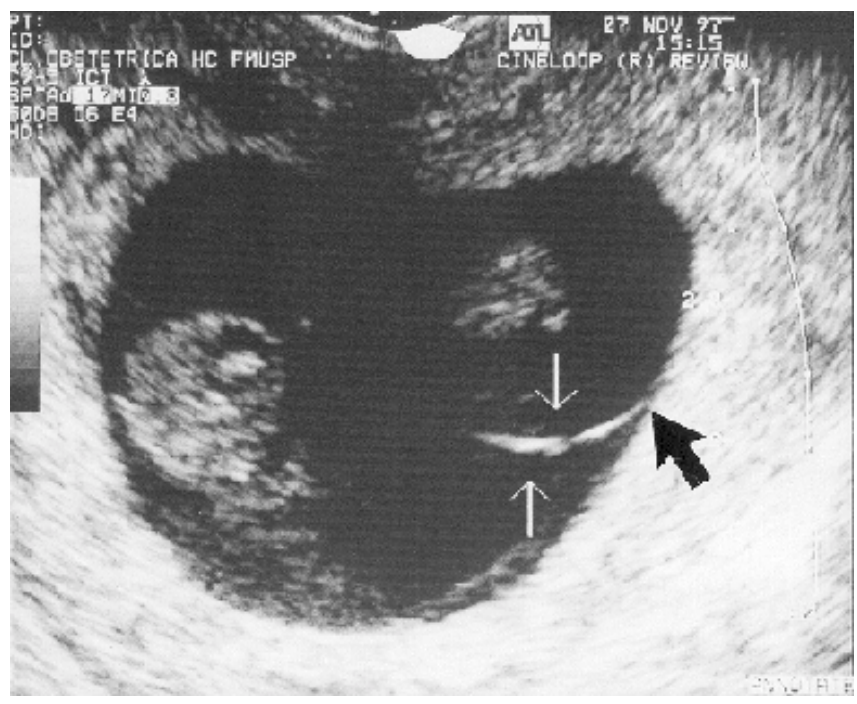

Figura 2 - Massa placentária única. A membrana amniótica se insere de forma abrupta no córion (seta; ausência do sinal de lamda), o que classifica a gestação como monocoriônica.

Tabela 1 - Distribuição das gestações mútiplas provenientes de fertilização espontânea.

\begin{tabular}{lc}
\hline Tipo de gestação múltipla & Fertilização espontânea (\%) \\
\hline Dupla & $89 \%$ \\
Tripla & $40 \%$ \\
Quádrupla & $50 \%$ \\
\hline
\end{tabular}

Em relação às gestações duplas ou gemelares, 43\% eram dicoriônicas, 26\% monocoriôni- 
cas e em $32 \%$ dos casos não foi possivel definir a corionicidade devido ao primeiro exame ultrasonográfico tardio ou parto em outro serviço ou ainda exame anatomopatológico da placenta não-realizado.

A idade gestacional média do primeiro exame ultra-sonográfico foi de 25 semanas (variação de 7-39 semanas), sendo que em $25 \%$ dos casos a primeira avaliação foi anterior à $14^{\mathrm{a}}$ semana de gestação.

A média de idade das pacientes foi de 30 anos, variando de 16 a 44 anos, sendo que $21 \%$ apresentavam idade igual ou superior a 35 anos. Em relação à cor, $44 \%$ eram brancas e $56 \%$ nãobrancas. Quanto a paridade, $42 \%$ eram nulíparas e $54 \%$ apresentaram partos prévios. $20 \%$ das gestantes eram portadoras de alguma patologia materna, como hipertensão, diabetes, tiroidopatias, cardiopatias, etc.

Do total de gestações $(n=169), 24$ apresentavam malformações fetais $(14,2 \%)$, cujo di- agnóstico foi realizado por ultra-sonografia antenatal. Em 121 gestações duplas em que se obteve o resultado perinatal, diagnosticaram-se $22(18,1 \%)$ com malformações fetais, sendo sete de ocorrência exclusiva em gêmeos (gêmeos unidos $n=4$; feto acárdico $n=3$ ) e 15 malformações fetais não-exclusivas para gêmeos. A distribuição das malformações fetais em relação à corionicidade está descrita na Tabela 2. Em relação às 10 gestações triplas, $2(20 \%)$ apresentavam malformações fetais (Tabela 3 ).

Tabela 2-Distribuição das malformações fetais em relaçãoà corionicidade de gestações gemelares.

\begin{tabular}{lc}
\hline Tipo de corionicidade & Malformações fetais \\
\hline Monocoriônica $(n=39)$ & $13(33 \%)$ \\
Dicoriônica $(n=58)$ & $4(7 \%)$ \\
Não-conhecida $(n=24)$ & $5(21 \%)$ \\
\hline$n=$ número de casos &
\end{tabular}

Tabela 3 - Distribuição das malformações fetais e tipo de amnionicidade encontrada nas 2 gestações triplas.

\begin{tabular}{lccc}
\hline Tipo de malformações fetais & Número de gestações afetadas & Número de fetos afetados & Corionicidade e amnionicidade \\
\hline Gêmeos unidos & 1 & 2 & Monocoriônica e monoamniótica \\
Síndrome da regressão caudal & 1 & 2 & Dicoriônica e triamnióticas
\end{tabular}

Gestações monoamnióticas foram observadas em 7 casos das gestações gemelares $(18 \%$ das gestações monocoriônicas) e em uma gestação tripla. Nas três gestações gemelares com os dois fetos separados, foi observado um caso de anomalia discordante entre os dois fetos e isto resultou em óbito neonatal do feto portador de cardiopatia complexa, após parto com 32 semanas, o recém-nascido normal sobreviveu. Um caso evoluiu para aborto espontâneo dos dois fetos com 12 semanas de gestação. No terceiro caso em que os dois fetos eram normais, os dois sobreviveram após parto com 34 semanas de gestação. $\mathrm{Na}$ gestação tripla monoamniótica os fetos unidos evoluíram com óbito neonatal e o feto normal sobreviveu após parto com 36 semanas.

O tipo de malformação fetal e tipo de amnionicidade encontrada nas 39 gestações gemelares monocoriônicas estão descritas na Tabela 4. O mesmo está descrito para gestações triplas na Tabela 3. As malformações fetais encontradas em 4 gestações dicoriônicas foram: malformação adenomatóide cística, espinha bífida, malformações do trato gastrointestinal e hidropisia não-imune. Em todos os casos somente 1 feto do par foi afetado.

Tabela 4 - Distribuição das malformações fetais e tipo de amnionicidade encontrada nas gestações gemelares monocoriônicas.

\begin{tabular}{lccc}
\hline Tipo de malformação fetal & Número de gestações afetadas & Número de fetos afetados & Amnionicidade \\
\hline Gêmeos unidos & 4 & 8 & Monoamnióticas \\
Gêmeo acárdico & 3 & 3 & Diamnióticas \\
Anomalia do trato gastrointestinal & 3 & 3 & Diamnióticas \\
Anomalia cardíaca & 2 & 2 & 1 diamniótica; \\
Higroma cístico & 1 & 1 & 1 monoamniótica \\
Total & 13 & 17 & Diamniótica \\
\hline
\end{tabular}


Em 5 gestações gemelares em que não foi possivel definir a corionicidade, foram encontradas as malformações fetais descritas na Tabela 5 .

\begin{tabular}{|c|c|c|}
\hline Tipo de malformações fetais & $\begin{array}{c}\text { Número de } \\
\text { gestações afetadas }\end{array}$ & $\begin{array}{l}\text { Número de } \\
\text { fetos afetados }\end{array}$ \\
\hline Displasia esquelética & 2 & 4 \\
\hline Síndrome da banda amniótica & 1 & 1 \\
\hline Anomalia do trato gastrointestinal & 1 & 1 \\
\hline CIUR e ventriculomegalia & 1 & 1 \\
\hline Total & 5 & 7 \\
\hline
\end{tabular}

CIUR = crescimento intra-uterino retardado simétrico
Em 152 gestações gemelares em que foi possivel definir a corionicidade, o peso de nascimento variou de $630 \mathrm{~g}$ a $3.050 \mathrm{~g}$ (média $1.969 \mathrm{~g}$ ) em 48 recém-nascidos gemelares monocoriônicos, e variou de $650 \mathrm{~g}$ a $3.480 \mathrm{~g}$ (média $2.337 \mathrm{~g}$ ) em 104 recém-nascidos gemelares dicoriônicos.

A diferença média de peso no nascimento entre os fetos de gestações monocoriônicas foi 377 g (40-1.070 g) e em gestações dicoriônicas foi $336 \mathrm{~g}$ (20-1.260 g).

A idade gestacional do parto em relação aos grupos monocoriônicos e dicoriônicos em relação à presença de malformações fetais está descrita na Tabela 6.

Tabela 6 - Distribuição do tipo de gestação gemelar (corionicidade) e a idade gestacional no parto.

\begin{tabular}{lcccc}
\hline $\begin{array}{l}\text { Corionicidade (sem e } \\
\text { com anomalias fetais) }\end{array}$ & Número de gestações & Média (semanas) & $\begin{array}{c}\text { Idade gestacional no parto (semanas) } \\
<32 \text { semanas }\end{array}$ & $<37$ semanas \\
\hline Monocoriônico & & 34 & $20 \%$ & $83 \%$ \\
sem anomalia & 39 & 35 & $10 \%$ & $76 \%$ \\
com anomalia & 26 & 32 & $39 \%$ & $94 \%$ \\
Dicoriônico & 13 & 37 & $11 \%$ & $50 \%$ \\
sem anomalia & 58 & 37 & $4 \%$ & $47 \%$ \\
com anomalia & 54 & 30 & $57 \%$ & $71 \%$ \\
\hline
\end{tabular}

\section{Discussão}

As anomalias fetais em gêmeos são divididas em duas categorias: malformações do tipo que também ocorrem em gestações únicas e aquelas associadas exclusivamente a gestações múltiplas. Esta última categoria inclui gêmeos unidos e gêmeos acárdicos. Kohl and Casey ${ }^{5}$ referem aproximadamente $2 \%$ de malformações maiores em crianças gêmeas e $1 \%$ em crianças provenientes de gestações únicas. A freqüência de malformações menores foi de aproximadamente $4 \%$ em gêmeos e 2,5\% em únicos ${ }^{5}$. Defeitos do tubo neural, hidrocefalia, malformações cardiacas, malformações do trato urogenital, anormalidades cromossômicas e artéria umbilical única ocorrem mais freqüentemente em gêmeos $^{4}$.

Rodis et al. ${ }^{6}$, em revisão de literatura sobre as anormalidades cromossômicas em gestações gemelares, concluíram que há evidência de aumento da incidência de aneuploidia.

Uchida et al. ${ }^{7}$ encontraram, em 29 abortos de gestações gemelares, 5 com anomalias cromossômicas $(17 \%)$, aproximadamente três vezes a taxa de ocorrência em gestações únicas.

Neste estudo a incidência de malformações fetais em gestações gemelares foi muito elevada $(14,2 \%)$ pois o Departamento de Obstetrícia do Hospital das Clínicas da Faculdade de Medicina da Universidade de São Paulo constitui serviço terciário e, portanto, de referência para gestações de alto risco.

A ultra-sonografia é essencial tanto para o diagnóstico como para a conduta em face de complicações perinatais comuns em gêmeos, incluindo anomalias fetais, gêmeos unidos, gêmeo acárdico, óbito fetal, prematuridade, crescimento intra-uterino retardado e sindrome da transfusão fetofetal. Todas as anomalias em gêmeos da população estudada foram diagnosticadas durante o pré-natal. Isto reflete a conduta do serviço, que recomenda acompanhamento ultrasonográfico detalhado e freqüente (a cada 4 semanas), durante o pré-natal, para todos os casos de gestação múltipla.

Há diferenças biológicas entre a gestação 
gemelar dizigótica e monozigótica. A gestação monozigótica ocorre de modo aleatório, resultante de um atraso na divisão, associada a fatores nutricionais, hipóxicos ou a algum outro agravo. Este atraso origina geralmente a placenta monocoriônica. Está hipótese pode explicar a alta incidência de malformações congênitas em gêmeos monozigóticos, ou seja, desde que a gestação monozigótica é resultado de um evento teratogênico, malformações associadas são mais frequentes que em gestações únicas ou dizigóticas $^{5}$. De outra parte, o risco de malformações, tal qual o risco de gestações gemelares dizigóticas, aumenta com o avanço da idade materna. Portanto, o aumento da taxa de malformações em gêmeos dizigóticos também é esperado.

Schinzel et al. ${ }^{8}$ dividiram estas malformações em 3 categorias. A primeira inclui aqueles defeitos resultantes de eventos teratogênicos (gêmeos unidos, sirenomelia, holoprosencefalia, defeitos de tubo neural e anencefalia). A segunda categoria resulta de conexões vasculares entre os gêmeos monozigóticos que podem levar a fluxo reverso, originando o feto acárdico. A morte de um feto intra-útero, com possível embolização por meio de anastomoses vasculares e isquemia regional no feto sobrevivente, pode ser responsável por defeitos como a aplasia cutis, rim em ferradura, hidrocefalia, porencefalia, encefalomalácea multicística, atresia intestinal, amputação de membros e microssomia hemifacial ${ }^{4,9,10}$. A última categoria é dos defeitos resultantes do crescimento intrauterino no final da gestação, crescimento discordante e posições adversas que afetam o fenótipo e podem resultar em alguns defeitos, como pé torto ou luxação congênita de quadril.

Gêmeos monozigóticos, freqüentemente, são discordantes para as anomalias, sendo que a taxa de concordância é baixa, variando de 9 a $18 \%$, pois não são necessariamente idênticos geneticamente nem o ambiente intra-uterino é sempre uniforme $e^{4,11}$. Gêmeos monozigóticos, embora raro, podem ter cariótipos diferentes (gêmeos heterocariotípicos) presumivelmente por resultado de não-disjunção pós-zigótica ${ }^{4}$. Há relato de que alguns defeitos estruturais discordantes em gêmeos monozigóticos podem ser resultado de alterações vasculares devido à divisão de fluxo circulatório ${ }^{9}$.

Neste estudo, anomalias acometendo os dois fetos foram observadas nos casos de gêmeos unidos e em um caso de gestação tripla dicoriônica e triamniótica, no qual o par de gêmeo monocoriônico apresentava anomalia concordante do tipo regressão caudal.
A placentação monocoriônica implica um ambiente menos favorável para o desenvolvimento do feto. Gêmeos monocoriônicos têm riscos aumentados, quando comparados a gêmeos dicoriônicos, sendo a mortalidade perinatal de $26 \%$ contra $9 \%$, respectivamente ${ }^{12}$. Neste estudo, a maioria das malformações fetais de gestações gemelares ocorreu em gestações monocoriônicas $(33 \%)$.

Gêmeos monoamnióticos são encontrados em cerca de $1 \%$ de todas as gestações gemelares ou em $5 \%$ dos gêmeos monocoriônicos. No presente estudo observamos $18 \%$ de gestações monoamnióticas, e disto decorre o número de gêmeos unidos presentes nesta série. Gêmeos unidos ocorrem somente uma vez em cada 50.000 partos ${ }^{13}$. A divisão da massa embrionária, após o $12^{\circ}$ dia de fertilização, resulta em gêmeos unidos, encontrados em cerca de $1 \%$ das gestações monocoriônicas. Os gêmeos unidos são classificados, de acordo com o local do corpo onde a união fetal é dominante, em cinco tipos predominantes: toracópagos (tórax, 30-40\%), onfalópagos (abdome, 25-30\%), piópagos (sacro, 10-20\%), isquiópagos (pelve 6-20\%) e craniópagos (cabeça, 2-16\%). O diagnóstico ultra-sonográfico prénatal desta anomalia foi relatado primeiramente em $1977^{14}$. Desde este relato, numerosos casos têm sido descritos na literatura ${ }^{15-17}$. O prognóstico depende do local e extensão da união, mas, em geral, cerca de $50 \%$ são natimortos e um terço dos que nascem vivos apresentam defeitos graves, para os quais a cirurgia não é possivel. Nos casos dos nascidos vivos, em que é realizada a cirurgia planejada, cerca de $60 \%$ sobrevivem. Na presente série observamos 4 gestações gemelares e 1 gestação tripla com gêmeos únidos. Todos eram toracópagos com cardiopatia complexa e, com isso, os recém-nascidos evoluíram para óbito neonatal antes de serem submetidos à separação cirúrgica.

No presente estudo diagnosticamos três gestações gemelares monocoriônicas diamnióticas com gêmeo acárdico. Estima-se que o gêmeo acárdico ocorra aproximadamente em 1 para cada 30.000 a 35.000 partos $^{18,19}$. Há evidência de que o risco de acardia é consideravelmente alto em gestação monoamniótica ${ }^{20}$. As teorias sobre o mecanismo pelo qual se desenvolve a acardia são: falha no desenvolvimento cardíaco primário ou o coração estava presente mas sofreu atrofia, como resultado de perfusão passiva e circulação reversa (TRAP sequence- seqüência da perfusão arterial reversa em gêmeos). Os gêmeos, potencialmente normais, apresentam alta taxa de mortalidade devido à combinação de insuficiência cardíaca intra-uterina e prematuridade ${ }^{21}$. 
O tratamento pré-natal se faz por meio da oclusão do fluxo para o gêmeo acárdico, utilizando ligação endoscópica ou coagulação a laser do cordão umbilical

Houve diferença significativa quanto ao peso médio de nascimento entre gestações gemelares monocoriônicas e dicoriônicas, porém não ocorreu o mesmo em relação à diferença relativa entre o par de gêmeos nos grupos estudados. Apesar de a etiologia do crescimento discordante ou retardo de crescimento de um ou dois membros do par poder ser a mesma que o retardo de crescimento de únicos (insuficiência placentária, infecção em gestação precoce, anomalias estruturais e cromossômicas ou constitucional), este ocorre mais freqüentemente em gestações monocoriônicas, devido à possibilidade de ocorrência de síndrome da transfusão fetofetal.

Neste estudo, a idade gestacional quando do parto foi significativamente menor em gêmeos complicados por anomalias, comparada com os gêmeos sem anomalias. A alta taxa de mortalidade perinatal, para gestações gemelares anômalas, reflete a letalidade destas anomalias e a alta incidência de complicações decorrentes da prematuridade. A presença de anormalidade de um gêmeo do par aumenta significativamente o risco de parto pré-termo, comparado com gestações gemelares sem anomalias.

O óbito de um dos fetos, em gestações dicoriônicas, expõe o feto sobrevivente principalmente ao risco de parto pré-termo, que pode ser devido à liberação de citocinas e prostaglandinas, durante a absorção do tecido placentário morto. Em gêmeos dicoriônicos, o risco de óbito ou retardo mental do outro feto, em tais casos, é de $5-10 \%$, ao passo que em gêmeos monocoriônicos, o risco de óbito ou retardo mental é de pelo menos $25 \%$, em razão de episódios hipotensivos somados ao risco de parto prematuro. A hipotensão aguda é o resultado da hemorragia do feto vivo para a unidade fetoplacentária do feto morto. Em gestações únicas, o óbito e a retenção do feto podem estar associados com coagulação intravascular disseminada materna. Entretanto, em gestações gemelares com um feto morto, tal complicação raramente foi relatada.

Assim, a determinação precoce do tipo de placentação por meio da ultra-sonografia no $1^{\circ}$ trimestre é útil no estabelecimento do prognóstico e na conduta para as gestações gemelares.

\section{SUMMARY}

Purpose: to demonstrate the types of fetal malformations in multiple pregnancy and their relation to chorionicity. Methods: one hundred and sixty-nine multiple pregnancies were evaluated. In all cases prenatal ultrasound examination was performed during antenatal care. Chorionicity was defined by: first trimester ultrasound evaluation (absence of lambda sign); presence of two separate placentas; different fetal sex; pathological placental examination.

Results: twenty-four (14.2\%) fetal malformations were observed, 22 in twin and 2 in triplet pregnancy. In the group with fetal malformations 13 were monochorionic, 4 dichorionic and in 5 the chorionicity was unknown. Some malformations were unique to twins (conjoined twins $n=$ 5 , acardiac twin $n=3$ ) and others were nonunique to twins. The gestational age at delivery was lower in the group with fetal malformations compared to the group without fetal malformations.

Conclusion: the majority of malformations occurred in the monochorionic pregnancies. In multiple pregnancies early determination of chorionicity is helpful to establish the prognosis and to plan the management of pregnancy.

KEY WORDS: Multiple pregnancy. Fetal malformations. Chorionicity. Ultrasound. Placenta.

\section{Referências}

1. Wenstrom KD, Gall SA. Incidence, morbidity and mortality, and diagnosis of twin gestations. Clin Perinatol 1988; 15:1-11.

2. Malone FD, Craigo SD, Chelmow D, D'Alton ME. Outcome of twin gestations complicated by a single anomalous fetus. Obstet Gynecol 1996; $88: 1-5$.

3. Onyskowová Z, Dolezal A, Jedlicka V. The frequency and the character of malformations in multiple birth. J Eur Teratol Soc 1971; 4:496.

4. Little J, Bryan E. Congenital anomalies in twins. Semin Perinatol 1986; 10:50-64.

5. Kohl SG, Casey G. Twin gestation. Mt Sinai J Med 1975; 42:523-39.

6. Rodis JF, Egan JF, Craffey A, Ciarleglio L, Greenstein RM, Scorza WE. Calculated risk of chromosomal abnormalities in twin gestations. Obstet Gynecol 1990; 76:1037-41.

7. Uchida IA, Freeman VC, Gedeon M. Twinning rate in spontaneous abortions. Am J Hum Genet 1983; 35:987-93. 
8. Schinzel AA, Smith DW, Miller JR. Monozygotic twinning and structural defects. J Pediatr 1979; 95:921-30.

9. Hoyme HE, Higginbottom MC, Jones KL. Vascular etiology of disruptive structural defects in monozygotic twins. Pediatrics 1981; 67:288-91.

10.Mannino FL, Jones KL, Benirschke K. Congenital skin defects and fetus papyraceus. J Pediatr 1977; 91:559-64.

11.Fenner A, Malm T, Kusserow U. Intrauterine growth of twins. A retrospective analysis. Eur J Pediatr 1980; 133:119-21.

12.Burke MS. Single fetal demise in twin gestation. Clin Obstet Gynecol 1990; 33:69-78.

13. Hanson JW. Incidence of conjoined twinning. Lancet $1975 ; 2: 1257$.

14.Wilson RL, Shaub MS, Cetrulo CJ. The antepartum findings of conjoined twins. J Clin Ultrasound $1977 ; 5: 35-9$.

15.Austin E, Schifrin BS, Pomerance JJ, Gans SL, Komaiko MS. The antepartum diagnosis of conjoined twins. J Pediatr Surg 1980; 15:332-4.
16.Fagan CJ. Antepartum diagnosis of conjoined twins by ultrasonography. AJR Am J Roentgenol 1977; 129:921-2.

17.Gore RM, Filly RA, Parer JT. Sonographic antepartum diagnosis of conjoined twins. Its impact on obstetric management. JAMA 1982; 247:3351-3.

18.Gillim DL, Hendricks CH. Holoacardius: review of the literature and case report. Obstet Gynecol 1953; 2:647-53.

19.Napolitani FD, Schreiber L. The acardiac monster: a review of the world literature and presentation of two cases. Am J Obstet Gynecol 1960; 80:5829.

20.James WH. A note on the epidemiology of acardiac monsters. Teratology 1977; 16:211-6.

21.Van Allen MI, Smith DW, Shepard TH. Twin reversed arterial perfusion (TRAP) sequence: a study of 14 twin pregnancies with acardius. Semin Perinatol 1983; 7:285-93.

\section{$1^{0}$ CONGRESSO BRASILEIRO SOBRE MATERNIDADE SEGURA E SAÚDE REPRODUTIVA}

\section{Período: 15 a 18 Novembro de 2000}

\section{Local: Brasília}

\section{ORGANIZAÇÃO: SGOB}

\section{Tel.: (61) 245-3681}

